\title{
Management considerations in the treatment of migraine in adolescents
}

This article was published in the following Dove Press journal:

Adolescent Health, Medicine and Therapeutics

26 May 2010

Number of times this article has been viewed

\section{Christine A Matarese \\ Kenneth J Mack}

Mayo Clinic, Division of Child and Adolescent Neurology, Rochester, MN, USA
Correspondence: Kenneth J Mack Mayo Clinic, Division of Child and Adolescent Neurology, 200 First St SW, Rochester, MN 55905, USA

$\mathrm{Tel}+\mid 507284335$ I

Fax + I 5072840727

Email mack.kenneth@mayo.edu
Abstract: Migraine is common in adolescents. It can significantly reduce quality of life, may contribute to significant school absences, and disrupt social activities. This article will address the clinical presentation, natural history, types, evaluation, diagnosis and prognosis of migraine. Common adolescent lifestyle factors such as stress, irregular mealtimes, and sleep deprivation may exacerbate migraines. Management options are discussed including lifestyle modifications, acute and preventative therapies. Features of chronic daily headache including comorbid conditions, management, and outcome are also addressed.

Keywords: adolescent, migraine, chronic daily headache, management

Migraine is common in adolescents and can significantly reduce quality of life. The pain is intense, and often the patient cannot concentrate or function effectively during or immediately after episodes. Migraine may contribute to significant school absences and disruption of social activities. The burden of migraine may also cause emotional changes such as anxiety or sadness. ${ }^{1}$ Common adolescent lifestyle factors such as stress, irregular mealtimes, and sleep deprivation may exacerbate migraines. Medication compliance and lifestyle modification may be challenging in this age group. All of these issues deserve consideration in the management of adolescent patients with migraine. The appropriate diagnosis and treatment of our patients with migraine can improve their quality of life significantly.

\section{Clinical presentation and natural history of migraine}

The clinical presentation of migraine varies with age. While migraine is commonly thought to primarily affect adolescents and adults, it may begin in infancy or childhood. Migraine occurs in 2\%-5\% of preschool children, $10 \%$ of school-aged children and $20 \%-30 \%$ of teenage girls. ${ }^{2,3}$ Most migraineurs begin to experience their attacks when younger than 20 years and approximately $20 \%$ of migraine patients experience their first attack when younger than 5 years. ${ }^{4}$

In preschool children, migraine often consists of episodes involving an ill appearance, complaints of abdominal pain, vomiting, and the desire to sleep. These children may demonstrate pain by irritability, crying, rocking, or seeking a dark room in which to sleep. Five- to 10-year-old children with migraine often experience bilateral frontal, bilateral temporal or retro-orbital headache associated with nausea, abdominal cramping, vomiting, photophobia, phonophobia, and a need to sleep.

submit your manuscript $\mid$ www.dovepress.com 
Table I Common clinical presentation of migraine by patient age

\begin{tabular}{|c|c|}
\hline Age & Presentation \\
\hline Preschool-aged child & $\begin{array}{l}\text { - Ill appearing } \\
\text { - Abdominal pain/nausea/vomiting } \\
\text { - Desire to sleep }\end{array}$ \\
\hline Elementary school-aged child & $\begin{array}{l}\text { - Ill appearing } \\
\text { - Bilateral headache } \\
\text { - Abdominal pain/nausea/vomiting } \\
\text { - Photophobia/phonophobia } \\
\text { - Desire to sleep }\end{array}$ \\
\hline Middle school-aged child & $\begin{array}{l}\text { - } \pm \text { visual aura (age }>8 \text { years) } \\
\text { - Unilateral headache } \\
\text { - Photophobia/phonophobia/osmophobia } \\
\text { - Desire to sleep }\end{array}$ \\
\hline Adolescents and adults & $\begin{array}{l}\text { - Severe, unilateral throbbing headache } \\
\text { - } \pm \text { visual aura } \\
\text { - Nausea/vomiting } \\
\text { - Photophobia/phonophobia/osmophobia } \\
\text { - Desire to sleep }\end{array}$ \\
\hline
\end{tabular}

Parents frequently describe these children as ill appearing, pale, or with dark circles under their eyes. Migraine aura is an infrequent feature prior to the age of 8 years. Older children with migraine tend to present with a unilateral, temporal headache and may avoid bright lights, loud noises, and strong odors. Migraine relief typically is associated with sleep. The headache location and intensity often change within or between attacks. Adolescents and adults may experience more typical migraine features including severe, unilateral, throbbing pain. Associated features may include visual aura, photophobia, phonophobia, osmophobia, nausea, and vomiting. Exacerbating or triggering factors may include physical exertion, altered sleep schedule, missing meals, relative dehydration, stress, or menses. Changes in the weather have also been mentioned by patients as a migraine trigger. ${ }^{5}$

Migraine can occur with or without aura. Only $10 \%-20 \%$ of children with migraine experience an aura, often for the first time after the age of 8 years. The aura usually precedes the headache by less than 30 minutes and lasts for 5-20 minutes. The aura may present without headache. Children often are unaware or unable to describe their aura. The visual aura is the most common form in children, consisting of blurred vision, fortification spectra (zigzag lines), scotomata (field defects), scintillations, black dots, kaleidoscopic patterns of various colors, micropsia, macropsia (distortion of size), and metamorphopsia ("Alice in Wonderland" syndrome). Visual auras often are reported as moving or changing shapes; other auras include attention loss, confusion, amnesia, agitation, aphasia, ataxia, dizziness, vertigo, paresthesia, or hemiparesis. Aura symptoms vary widely within and between attacks. ${ }^{6}$

The actual headache phase of the migraine attack usually is shorter in the pediatric population compared with adults; pediatric headaches can last 60 minutes to 48 hours, but they usually last less than 4 hours. The severity of childhood headache often is milder than adult migraines. The headache phase often is associated with cold extremities, nausea, anorexia, vomiting, diarrhea or constipation, dizziness, chills, excessive sweating, ataxia, numbness, photophobia, phonophobia, memory loss, or confusion. After the headache phase, the patient may feel either elated and energized or more typically exhausted and lethargic. This stage of migraine may last from hours to days.

Migraine is associated with a variety of co-morbid conditions. Psychiatric symptoms such as depression, panic attacks, anxiety disorders or phobias may be present. Some patients may not meet full Diagnostic and Statistical Manual for Mental Disorders (4th edition; DSM-IV) criteria for these disorders. ${ }^{7}$ However other patients will meet DSM-IV criteria for psychiatric disorders. One recent study found that after controlling for age and sex, those with migraine have a 2.6-times greater prevalence of major depression than those without migraines. This was increased compared to other chronic medical conditions and the association persisted into the older age groups as well. ${ }^{8}$ Other psychiatric symptoms are seen, in addition to migraine. A large population based study on the comorbidity of migraine and psychiatric disorders found that major depressive disorder, bipolar disorder, panic disorder, and social phobia occurred more than twice as often in patients with migraine than those without and that this was not related to socioeconomic variables. ${ }^{9}$ Epilepsy and migraine often occur within the same individual, although most patients with migraines do not have seizures. ${ }^{10}$ Migraineurs are more prone to motion sickness than patients without migraine. ${ }^{11}$ Intermittent vertigo is found frequently in patients with migraine. ${ }^{12}$ There is a higher cardiovascular reactivity to postural changes in patients with migraine and this may result in dizziness. ${ }^{13}$ Migraines are associated with sleep disturbances in some patients, but this may be secondary to other comorbid factors. ${ }^{14}$ Ingestion of ice cream caused headache in $93 \%$ of migraine subjects. The headache typically is located at the usual site of migraine pain. ${ }^{15}$

\section{Types of migraine}

A patient may experience varying types of headaches, including different forms of migraine. The two most frequent 
forms are migraine with or without aura. Migraine variants also exist. Status migrainosus is a severe form of migraine in which the headache attack is continuous over 72 hours. Patients usually have a pre-existing migraine history.

Familial hemiplegic migraine is an autosomal-dominant form of migraine with aura. ${ }^{16}$ Patients have a prolonged hemiplegia that can be accompanied by numbness, aphasia, and confusion. The hemiplegia may precede, accompany, or follow the headache and symptoms may last for hours to days. The headache usually is contralateral to the hemiparesis. Some familial hemiplegic migraine is associated with cerebellar ataxia. Other types of severe familial hemiplegic migraine may present with coma, fever, and meningismus.

Basilar-type migraine is a subtype of migraine with aura, and is observed mostly in adolescent and young adult females. Headache pain may be located in the occipital area. Basilar-type migraine is characterized by disturbances in function believed originating from the brain stem, occipital cortex, and cerebellum. The occipital headache must have at least two of the following aura symptoms: dysarthria, vertigo, tinnitus, hyperacusia, diplopia, bi-field visual symptoms, ataxia, decreased level of consciousness or bilateral paresthesias. A history of typical migraine exists in many families. Some patients experience basilar migraine attacks intermingled with typical migraine attacks. ${ }^{17}$

Some migraine variants are typically seen in the younger child. As the patient gets older the more characteristic migraine episodes may develop. Examples include benign paroxysmal vertigo, acute confusional migraine, cyclic vomiting, abdominal migraine, paroxysmal torticollis and acephalgic migraine of childhood. A prior history of these disorders is an important factor when obtaining the headache history.

Benign paroxysmal vertigo of childhood is a condition characterized by brief episodes of vertigo, disequilibrium, and nausea, usually found in children aged 2-6 years. The patient may have nystagmus within but not between the attacks. The child does not have hearing loss, tinnitus, or loss of consciousness. Symptoms usually last only a few minutes. These children often develop a more common form of migraine as they mature.

Acute confusional migraine is characterized by transient episodes of amnesia, acute confusion, agitation, lethargy, and dysphasia. This form of migraine is often precipitated by minor head trauma. The child may have a receptive or expressive aphasia, and the confusional state may either precede or follow the headache. Some children also experience recurrent episodes of confusion. The patient usually recovers within hours. The child may not have a history of headache but usually develops typical migraine attacks when older.

Migraine-associated cyclic vomiting syndrome is characterized by recurrent periods of intense vomiting separated by symptom-free intervals. Many patients with cyclic vomiting have regular or cyclic patterns of illness. Symptoms usually have a rapid onset at night or in the early morning and last 6-48 hours. Associated symptoms include abdominal pain, nausea, retching, anorexia, pallor, lethargy, photophobia, phonophobia, and headache. The headache may not appear until the child is older. Migraine-associated cyclic vomiting syndrome usually begins when the patient is a toddler and resolves in adolescence or early adulthood; it rarely begins in adulthood. More females than males are affected by cyclic vomiting. Usually a family history of migraines in the parents or siblings is present. These children often experience severe fluid and electrolyte disturbances that require intravenous fluid therapy.

In abdominal migraine, the patient may suffer from recurrent bouts of generalized abdominal pain with nausea and vomiting, but often with no headache present. The episodes are often relieved by sleep and later the child awakens feeling better. Abdominal migraine may alternate with typical migraine and can lead to typical migraine as the child matures.

Paroxysmal torticollis of infancy is an uncommon disorder characterized by repeated episodes of head tilting associated with nausea, vomiting, and headache. Attacks usually occur in infants and may last from minutes to days. ${ }^{18}$ Posterior fossa abnormalities should be considered in the differential diagnosis. Data have linked these symptoms to mutations in the CACNA1A gene in some patients. ${ }^{19}$

Acephalgic migraine of childhood (migraine sine hemicrania) is characterized by a migraine aura without headache, usually visual auras, and a female predominance. A family history of migraine is frequent. In one series, this represented $2 \%$ of childhood migraineurs. ${ }^{20}$

\section{Evaluation and diagnosis of migraine}

There is no specific diagnostic test for migraine. The diagnosis is made through the history, physical exam, and clinical judgment. Obtaining a family history is important as you may uncover a family history of migraine or motion sickness. The adolescent with migraine should have a normal general physical examination and a normal detailed neurologic examination. 
Only a small percentage of headache patients require further laboratory and radiologic studies. An imaging study should be considered in patients with a history of seizures, recent head trauma, significant change in the headache, or evidence of focal neurologic deficits or papilledema upon physical examination. No absolute rules exist in the evaluation of the headache patient; the need for a neuroimaging study ultimately is based on clinical judgment. Electroencephalography is not useful in the routine evaluation of headache patients. It may be considered in patients with an atypical migraine aura, episodic loss of consciousness, or symptoms suggestive of a seizure disorder. Lumbar puncture is indicated if meningitis, encephalitis, subarachnoid hemorrhage, or high-low pressure syndromes are considered. Patients in whom elevated intracranial pressure is suggested or with focal neurologic deficits should undergo a neuroimaging study prior to a lumbar puncture.

\section{Management of migraine}

The management of migraine headaches should emphasize identification of environmental trigger factors, pain control at the time of the headache, and preventative medication.

The treatment of adolescents with mild, infrequent attacks consists primarily of rest, trigger avoidance, and analgesics. The first step in migraine treatment is to explain the disease to the patient and the parents. They will benefit from a simple explanation of the headache pain and reassurance that it is not caused by a brain tumor or other life-threatening conditions. Environmental and lifestyle factors such as regular sleep and meal schedules and avoidance of overloading the patient's schedule with activities are important. Helping the adolescent recognize migraine triggers is helpful. It is important that the patient has realistic expectations; identifying and avoiding triggers reduces the frequency of migraine headache but does not eliminate headaches.

Psychological triggers include stress, anxiety, worry, depression, and bereavement. Emphasizing to the adolescent and family members that migraine is not an imagined or psychological illness is important. Stress is not the sole cause of the headaches, although it makes migraines more difficult to manage. Physiological triggers include fever or illness, missing a meal, fatigue, and sleep deprivation. Environmental triggers of migraine include fluorescent light, bright light, flickering light, fatigue, barometric pressure changes, high altitude, strong odors, computer screens, or rapid temperature changes. Some patients report that complex visual patterns like stripes, checks, or zigzag lines may trigger migraines. Physical exertion can trigger migraine. Some migraineurs report that they are more likely to get a headache after participating in sports or being extremely active. Minor head trauma (eg, being hit in the head with a ball, falling on one's head) also may result in a migraine attack. Travel or motion may cause migraine, particularly in younger patients.

\section{Acute migraine management}

At the time of the headache, it is advisable that the patient lies down in a cool, dark, quiet room and fall asleep. Sleep is the most potent antimigraine treatment. Some patients find that ice or pressure on the affected area of pain can alleviate pain temporarily. Nonsteroidal anti-inflammatory agents (NSAIDs) are effective if taken at an appropriate dosage during the aura or early headache phase. ${ }^{21}$ Common side effects include gastrointestinal complaints and taking NSAIDS with meals may lessen this risk. Gastric stasis occurs in migraine patients and causes delay in absorption of oral medications. Occasionally, carbonated beverages may improve absorption. Nonpharmacologic treatment modalities such as self-relaxation, biofeedback, and self-hypnosis may be reasonable alternatives to pharmacologic treatment in managing migraine, particularly in adolescents. ${ }^{22}$

In terms of medications, many adolescent patients will benefit from the use of typical adult dosages of triptans. The use of triptans in younger children between the age of 6 and 12 years has also been studied, but requires a more careful titration of the dose to body size. Side effects can include worsening headache, paresthesias, and chest pain. ${ }^{21}$ Recently, the United States Food and Drug Administration (FDA) has approved the use of almotriptan, the first triptan approved for use in children under the age of 18 years, based in part on recent new data showing its safety and efficacy at this age. ${ }^{23}$

Excessive use of over-the-counter (OTC) pain medications and analgesics can cause occasional migraine attacks to convert to analgesic-abuse headaches or rebound headaches. Advise patients to avoid frequent, long-term use of NSAIDs, acetaminophen, triptans, or ergotamines.

\section{Preventative medications}

Prophylactic or preventative medications are taken on a daily basis to reduce the frequency or severity of headaches and associated symptoms. A good response to prophylactic medications is often considered a $50 \%$ reduction in the frequency or severity of attacks. Reserve consideration of prophylactic drugs for adolescents with frequent ( $>2$ per week), prolonged, and disabling migraine attacks that do not adequately respond to other treatments. Often, several weeks are necessary 
before therapeutic gains are observed with prophylactic medications. Some patients must be maintained on longterm prophylactic therapy, and others tolerate drug holidays, particularly during summer when migraines are less frequent for many adolescents. Possible preventative medications include amitriptyline, propranolol, gabapentin, valproate, topiramate, verapamil, and riboflavin. ${ }^{21,24}$ Decisions on which medication to chose can be assisted by patient comorbidities and medication side effect profiles. Amitriptyline can cause cardiac dysrhythmias, weight gain and fatigue. Propranolol may cause fatigue, dyspnea, bradycardia and depression. Gabapentin may cause peripheral edema and fatigue. Weight gain and gastrointestinal disturbances may be seen with valproate. Topiramate has a potential benefit of weight loss but may cause word finding difficulties and slowed psychomotor reactions which may be problematic in school aged patients. Additional adverse effects include paresthesias, decreased sweating and nephrolithiasis. Verapamil may cause hypotension and dizziness. Patients wishing to avoid prescription medications may be interested in trying riboflavin for headache prevention. It is a water soluble vitamin with few side effects.

Medication compliance should be a consideration in the adolescent age group. The adolescent should be actively involved in the decision on medication options. For example, some may prefer to try nonpharmacological therapies such as biofeedback first. Side effects of medications should be discussed as weight gain and cognitive slowing may be particularly important to adolescents. Consider using once daily dosing to improve compliance or discuss strategies to improve compliance such as planning to take the medication along with a pre-existing routine such as mealtime or teeth brushing. Reasonable expectations to benefits of medications should be discussed as should the prolonged time period often necessary to observe an improvement. Medications indicated for associated factors such as antiemetics for nausea and vomiting should also be discussed.

Evidence on the pharmacologic management of headaches in children and adolescents was reviewed by the American Academy of Neurology and the Child Neurology Society. A Practice Parameter was published on this topic in 2004. Ibuprofen, acetaminophen, sumatriptan, rizatriptan and zolmitriptan for acute headache treatment were reviewed. The committee's recommendations included effectiveness of ibuprofen and probable effectiveness of acetaminophen in the acute treatment of migraine in children and effectiveness of sumatriptan nasal spray for acute migraine treatment in adolescents. Prophylactic medications were also reviewed including divalproex sodium, topiramate, levetiracetam, trazodone, pizotifen, amitriptyline, cyproheptadine, flunarizine, nimodipine, propranolol, and clonidine. Their recommendations on the preventative therapy of migraine in children and adolescents included the probable effectiveness of flunarizine; however this is not available in the United States. They felt there was insufficient evidence to make any recommendations about the use of cyproheptadine, amitriptyline, divalproex sodium, topiramate or levetiracetam. They found conflicting evidence regarding propranolol and trazodone. They did not recommend the use of pizotifen or nimodipine as they did not demonstrate efficacy. ${ }^{21}$ Since that practice parameter, it has been shown in an intent-to-treat randomized controlled study that $100 \mathrm{mg}$ /day of topiramate was effective in reducing monthly migraine days, whereas $50 \mathrm{mg}$ /day was no different than placebo. ${ }^{25}$

\section{Prognosis of episodic migraine}

It is difficult to prognosticate the future in an individual migraine patient. In one of the few longitudinal studies of childhood migraine patients, children with migraine were followed for 40 years. ${ }^{4}$ The average onset of the migraines occurred at 6 years. During puberty or young adulthood, $62 \%$ of the children were migraine free for at least 2 years; $\sim 33 \%$ of these children regained regular attacks after an average of 6 migraine-free years, and a surprising $60 \%$ of the original 73 children still had migraines after 30 years; $22 \%$ of the subjects never had a migraine-free year. Of those patients who became parents, $52 \%$ have at least 1 child in their present or previous families who developed recurrent migrainous headache.

\section{Chronic daily headache}

Adolescents with episodic migraine may also develop chronic daily headache. This may be associated with frequent use of analgesic medications or occur following an illness. This entity shares some features with migraine but management strategies differ from those of episodic migraine.

Chronic daily headache is a disorder where the diagnosis is based on the presence of headache for greater than or equal to 15 headache days in 1 month, over a period of three consecutive months, and with no underlying organic pathology. ${ }^{17}$ The headaches last for more than 4 hours per day. This headache disorder tends to affect teenagers and adults, but can also occur in preteens. It can occur in up to $4 \%$ of young women and up to $2 \%$ of young men, with similar prevalence rates seen in studies from Asia, Europe, and the United States. ${ }^{26}$ Chronic daily headache has been shown to 
represent up to $60 \%$ of the cases seen in pediatric headache specialty clinics. ${ }^{27,28}$

Silberstein and others ${ }^{29,30}$ have defined 4 different categories of chronic daily headache based upon symptoms. These include transformed or chronic migraine, chronic tension type headache, new daily persistent headache, and hemicrania continua. Many teenage patients with chronic daily headache have a past history of episodic migraine. The transformation to a chronic migraine may occur over a period of weeks to months, or it may occur abruptly over a matter of hours. Approximately a quarter of teenagers with chronic daily headache will have no significant past headache history. In these latter patients, an infection such as mononucleosis or a minor head injury may incite a new daily persistent headache. A smaller number of patients will have a history of tension-type headaches prior to their chronic daily headache. ${ }^{31,32}$

Most commonly, the adolescent with chronic daily headache will complain of at least 2 types of headaches. ${ }^{27}$ Prominent are severe intermittent headaches that are migraine-like. They tend to be pan-cephalic or in the front of the head. The severe headaches will be described as throbbing, severe, crushing, knife-like, or hatchet-like. They are often associated with nausea during the most severe times, and the patient will frequently have photophobia, phonophobia, and osmophobia. For this more severe headache pain, sleep will sometimes help, but they will still have persistent headache when they awaken. The frequency of these severe headaches will vary with the individual. The severe episodes typically occur multiple times a week.

In addition to these severe intermittent headaches, the adolescent with chronic daily headache will often complain of a continuous headache that is present 24 hours a day, 7 days a week. This continuous headache may wax and wane in severity, often being worse either in the morning or at the end of the school day. The characteristics of the all-the-time headache pain are similar to the episodes of severe headaches, only much less intense. Some patients may also describe this all-the-time headache as having features of a tension type headache, with the pain being band-like or crushing rather than throbbing.

Headache is not the only symptom in chronic daily headache. Chronic daily headache is really a multiple symptom complex. Frequent comorbid symptoms include dizziness, sleep disturbance, pain at other sites of the body including neck pain, back pain, and abdominal pain, fatigue, difficulty in concentration, decreased mood, and increased anxiety.
It is important to recognize and treat these other symptoms as well.

Many patients will transition from a headache-free period or episodic migraines to chronic migraines during an infection. Consideration should be given to serology for Epstein-Barr virus, West Nile virus, and Lyme disease. Although for some of the viral etiologies there is no specific treatment, many of the patients appreciate knowing that there was a physiological underpinning for the transition to a chronic headache.

Perhaps the most useful role of the neuroimaging study in chronic daily headache is to reassure the adolescent and their family. On the other hand, an imaging study is more likely to be informative if there are focal deficits present on examination, papilledema, or a history of seizures. ${ }^{33}$ Often, a magnetic resonance imaging (MRI) of the head is performed; however, the MRI will be normal in the overwhelming majority of chronic daily headache patients. Occasionally in migraine patients, white matter abnormalities, arachnoid cysts, or pineal cysts will be seen that are generally believed to be of no clinical significance to the chronic daily headache. If a patient has had a significant history of head or neck trauma, particularly at the onset of the chronic daily headache, then an MR angiogram of the neck should also be considered to rule out a possible carotid dissection. When pseudotumor cerebri is a strong consideration, then an MR venogram should also be considered since sinus thrombosis can cause elevated intracranial pressure.

\section{Comorbid conditions in chronic daily headache}

Disability in adolescents can be measured in days with headaches, school absence, disruption of social activities, and family dysfunction as a result of headache. For research and clinical purposes there are validated tools to measure headache disability in adolescents. ${ }^{34}$

The patient may be habituated to OTC or prescribed medications. Combating rebound from those substances is part of the treatment. Medications implicated in this overuse syndrome include most OTC analgesics and decongestants, opioids, butalbital, isometheptene, benzodiazepines, ergotamine, and triptans. ${ }^{35}$

Sleep is disrupted in at least two thirds of the patients who have chronic daily headache. ${ }^{36}$ A common sleep disturbance is a delayed sleep onset and often times these individuals will not be able to fall asleep for 30 minutes to several hours after they go to bed. Some patients will wake frequently during the night as well. Occasionally one elicits a history of pain 
Table 2 Common comorbidities in migraine and chronic daily headache

\begin{tabular}{ll}
\hline Episodic migraine & - Anxiety/depression \\
& - Dizziness/vertigo \\
& - Sleep disturbance \\
& - Motion sickness \\
& - Ice cream headache \\
& - Epilepsy \\
& - Anxiety/depression \\
& - Dizziness/vertigo \\
Chronic daily headache & - Sleep disturbance \\
& - Fatigue \\
& - Diffuse or abdominal pain \\
& - Difficulty concentrating \\
\hline
\end{tabular}

in the legs and restless legs during the night. Consideration may be given to a formal sleep study since lack of sleep may be one factor that aggravates the headache symptoms. Typically the headache syndrome will not resolve until the sleep is improved.

Many chronic daily headache patients have symptoms of dizziness. The dizziness is associated with feeling weak and unsteady, and with changes (blurring or loss of) in vision. The dizziness is often positional, and they may experience syncope or near syncope several minutes after standing. There is typically no vertigo, except during severe headache episodes. The dizziness is particularly prominent in the morning after they first get up. During the office exam, a difference in blood pressure or pulse rate between sitting and standing may be noted. The patient often experiences mild symptoms of this dizziness if stood up for several minutes in the office. One may see either a significant tachycardia with standing (postural orthostatic tachycardia syndrome) and/or a decrease in the systolic blood pressure with standing. A tilt-table test will help confirm orthostatic hypotension, and these symptoms can be appropriately addressed and treated.

Mood problems and anxiety frequently coexist with chronic daily headache. The mood problems may precede or follow the onset of the headache. In some patients it is possible to resolve the problems with mood without affecting the headache, and in other patients it is possible to improve the headaches without improving the mood problem. Chronic daily headache should be considered a primary headache syndrome and not a mood disorder. Both the symptoms of headache and mood need to be addressed. If there are significant problems with mood and anxiety, it is difficult to control the headaches until these symptoms are improved.

Other frequent co-morbid symptoms include nonspecific abdominal pain, back pain, neck pain, and diffuse muscle and joint pain. Often no additional organic etiology is found to explain these additional pain symptoms. The longer the duration of the chronic daily headache syndrome, the more prominent these symptoms seem to become. In many patients, these additional areas of pain seem to be part of a much more diffuse pain syndrome.

\section{Management of chronic daily headache}

Chronic daily headache is difficult to control. There are often no immediate or easy answers to the treatment and resolution of the pain. Many of these patients have had a sudden onset to their daily headache, and some can even point to a specific day when they transitioned from a headache-free life to an all-the-time headache. Unfortunately, it typically takes weeks to months to effect a change in headache control. Similarly to episodic migraine management, the cornerstones of therapy are education, preventative medications, and attention to routine.

It is difficult for many families to comprehend that the head pain can persist for such a long time, that there are no abnormalities showing up on diagnostic testing, and that the medications prescribed are not immediately effective. It is not unusual for these patients to see multiple doctors or neurologists because of this frustration. To limit this frustration, it is useful to spend adequate time with the adolescent and their family in discussing chronic daily headache. The discussion should include describing what chronic daily headache is, how secondary causes of headache have been ruled out, the role of medications, when not to use pain relievers, the role of nonmedication approaches (such as biofeedback or physical therapy), and what can be expected in the short and long term.

Preventative medications are traditionally used in episodic migraines to reduce the frequency of the migraine headaches. However, in chronic daily headache, a reasonable therapeutic goal would be to make the severe intermittent headaches less frequent, and to make the all-the-time headache less intense. The most published experience of preventatives in chronic migraine or chronic daily headache is the use of medicines that work on the serotonergic system or the anticonvulsants. Unfortunately, there have been few prospective randomized controlled studies in pediatric patients to give us guidance as to what is the most effective or safe medication to use in chronic daily headache. ${ }^{21}$

Studies in adults and children have shown that tricyclic antidepressants, such as amitriptyline, are helpful in chronic daily headache. ${ }^{37} \mathrm{~A}$ key to success is to be willing to go up to higher therapeutic levels of these medications 
than is traditionally used in episodic childhood migraine. Consideration needs to be given to electrocardiogram changes, including an increased corrected QT interval. Weight gain is a significant concern in adolescent patients with these medicines, and it affects some patients more than others. Amitriptyline can also be helpful for sleep onset. Other tricyclics, such as nortriptyline or protriptyline, may cause less sedation.

Other serotonergic agents such as the selective serotonin reuptake inhibitors (SSRIs) have also shown to be effective in some adults with chronic headache. ${ }^{38}$ The SSRIs seem to be less effective than the tricyclics for pain control, although they are more helpful in children for their positive effects on mood. In select patients, the use of an SSRI can be very useful. Fluoxetine is the only FDA-approved SSRI for use in children, and it is metabolized through the same pathway as the tricyclics, so care should be given when using fluoxetine (or paroxetine) with the tricyclics at the same time, since that may cause unexpectedly high levels of both.

Studies in headache patients have shown anticonvulsants are also useful. Valproate, topiramate, and gabapentin have been used. ${ }^{21}$ Choice of rational pharmacotherapy to treat the patient's other problems is ideal. Antidepressants can address underlying mood disorders as well as sleep problems. Beta-blockers can make depression worse; however, they may be helpful for patients with a postural orthostatic tachycardia syndrome. Calcium channel blockers are useful for patients who also have hypertension, but cause constipation and orthostatic hypotension. If the patient needs to lose weight, topiramate is a good choice, although this may result in mental clouding. The use of botulinum toxin shows promise as well. ${ }^{39}$

Pain control at the time of the headache is a very difficult problem for patients. Analgesics that are typically effective for episodic migraine headaches are not very effective for chronic migraine or chronic daily headaches. Most patients report that pain relievers are not effective for the all-the-time, " $24 / 7$ " headache. It is reasonable to discourage patients from trying to use analgesics to treat the all-the-time headache, since this may result in analgesic overuse and a potential analgesic rebound headache. In contrast, for the more severe intermittent headache episodes with migrainous qualities, analgesics should be considered. Pain approaches can include the use of migraine pain relievers such as triptans, indomethacin or other NSAIDs. Compounds that contain caffeine, barbiturates, opiates, or that have a high potential for rebound should be limited or avoided. Patients typically find that when the preventative medication starts working, then the pain analgesics will become more effective also.
Some headache centers will aggressively try to treat the headache pain with intravenous protocols. Several approaches are used alone or in combination. Dihydroergotamine is effective for status migrainous, and some patients will get pain relief for their chronic headache pain. ${ }^{40}$ Valproate also comes in an intravenous (IV) formulation as well and has been used to abort severe headache episodes. ${ }^{41}$ Finally, some patients respond to a short-term use of oral steroids or IV steroids. These approaches seem to be most helpful for patients who have the recent onset of daily headaches. Many patients will feel better while hospitalized for these treatments, but may revert back to their typical headache after leaving the hospital. The hospitalization does provide the patient an opportunity for education about headaches, introduction to biofeedback, and in some cases physical therapy.

Nonpharmacological approaches to the headaches are also very important. Because of the chronic nature of the pain, some adolescents will benefit with a consultation with a psychologist to at least be introduced to the techniques of relaxation therapy and biofeedback. Mood problems and anxiety frequently coexist with chronic daily headache. ${ }^{42}$ The psychologist can address the issues of mood and anxiety.

Many of the patients have been ill for months to years and have become physically "deconditioned". Starting a reconditioning exercise program is very important. Patients should be encouraged to start slowly. For the most severely affected patients, begin with ten minutes of aerobic exercise a day and then increase the time by $10 \%$ a week. The key is to be slow but persistent in the increased activity level. Other nonpharmacological therapies that may also provide benefit include acupuncture, and massage therapy. A physical therapy consult can be helpful in these situations. ${ }^{43,44}$

Because the headache is daily, and often $24 / 7$, it is difficult to identify specific trigger factors for the chronic daily headache. However, there are important environmental factors that do play a role in these headaches. There is an interesting seasonal variability in the degree of chronic daily headache symptoms. Most adolescents will do better in the summertime, and frequently have a worsening of their headaches at the start of the school year. Therefore, September and October are particularly bad times for the worsening of headaches. In some patients, the need for a preventative medication is greatly reduced in the summertime, but increases again in the fall. The reason for this variability is unknown, but may relate to stress, loss of sleep, bright lights in the school, decreased access to exercise, decreased time for relaxation, and the propensity of some teenagers to skip breakfast to make it to school on time. 
School absence is a significant problem. Once patients have been out of school for a period of weeks, it is very difficult for them to return to a regular school schedule. Frequently patients will observe that after returning to 2 or 3 days of a full-day schedule, they come back home with a more severe headache. Some patients do best with a gradual reintroduction into the school system. These patients have more success returning to school on an abbreviated schedule, often with 1 or 2 class periods that are around the lunch hour. It is difficult for these patients to start off with early morning classes, since many of them have sleep disturbances and lack a full night's sleep.

\section{Outcome in chronic daily headache}

It is important to state the expectations of preventative therapy to the patient and the family. Preventative therapy may improve the headaches, but it will not eliminate the headaches in the short term. A common pattern observed during treatment is first, the severe migraine episodes become less frequent, then the all-the-time headaches start to improve and patients gradually experience the onset of headache-free days. After 1 month of an effective therapy, a reasonable expectation would be to have less frequent severe headache episodes, and a decrease in the intensity of the all the time, 24/7 headache. It is rare to see complete resolution of the headaches after a short period of time. Once a trend toward improvement is seen, the dose of medication is adjusted for optimal control of the headaches, and the patient is continued on the preventative for at least 6 months of good (but rarely complete) symptom control.

Follow-up should be scheduled on a routine basis until symptoms come under good control. It is not unusual to make frequent adjustments in management, and it may take months before matching up the right preventative medication or the right therapeutic approach with the individual patient. It is very difficult to work with these patients over the phone. Although the parents can describe the pain with a certain

Table 3 Counseling points in migraine and chronic daily headache

\begin{tabular}{l}
\hline Lifestyle modifications: \\
- Regular sleep schedule \\
- Regular mealtimes \\
- Adequate fluids \\
- Regular cardiovascular exercise \\
Avoidance of frequent analgesic use \\
Medication side effects \\
Expectations of treatment: \\
- Decrease in frequency of severe headaches \\
- Decrease in intensity of headaches \\
- Time frame for noticeable improvement \\
\hline
\end{tabular}

number over the phone, it is harder for the parents to describe the patients' affect, mood, function, and appearance over the phone. These target symptoms are just as valuable treatment indicators as their pain scale.

Adolescents should be aware that these symptoms wax and wane throughout the year. The vast majority of patients do better in the summertime when they have more access to sleep, they have less stress, and more physical activity in their daily lives. Unfortunately it is not unusual for many of these symptoms to rebound in September and October.

There are limited data looking at the outcome of chronic daily headache in children. ${ }^{31,42}$ It is not unusual to see patients who have chronic daily headaches persisting for months to years. Most patients will resolve their headache within 2 years, but some may have a chronic daily headache for eight years or more..$^{45}$ It is hoped that with successful identification of this syndrome and aggressive pharmacologic and nonpharmacologic management, the average duration of chronic daily headache in adolescents could be considerably less. When adolescents do show significant improvement, often times they will return to their premorbid state, which in essence is to become an episodic migraine patient.

\section{Conclusion}

Episodic migraine and chronic daily headache are common in the adolescent age group. These patients may have a prior history of a migraine variant, less severe occasional headaches or may have recently developed headaches. The head pain can be at best disruptive to the adolescent's lifestyle and at worst debilitating. Patients may have significantly altered their lifestyle because of their headaches and may experience significant co-morbidities. Various age related exacerbating factors should be addressed and the adolescent should be actively involved in the treatment plan. Multiple medication options are available and should be chosen based on comorbidities and side effects. Resolution is not immediate and compliance and lifestyle modification require effort on the part of the adolescent and their family. Appropriate diagnosis and treatment can improve quality of life significantly.

\section{Disclosures}

The authors report no conflict of interest in this work.

\section{References}

1. Powers SW, Patton SR, Hommel KA, Hershey AD. Quality of life in childhood migraines: clinical impact and comparison to other chronic illnesses. Pediatrics. 2003;112(1 Pt 1):e1-e5.

2. Abu-Arefeh I, Russell G. Prevalence of headache and migraine in schoolchildren. BMJ. 1994;309(6957):765-769.

3. Aromaa M, Sillanpaa ML, Rautava P, Helenius H. Childhood headache at school entry: a controlled clinical study. Neurology. 1998;50(6):1729-1736. 
4. Bille B. A 40-year follow-up of school children with migraine. Cephalalgia. 1997;17(4):488-491; discussion 487.

5. Mukamal KJ, Wellenius GA, Suh HH, Mittleman MA. Weather and air pollution as triggers of severe headaches. Neurology. 2009;72(10): 922-927.

6. Lendvai D, Crenca R, Verdecchia P, et al. Migraine with visual aura in developing age: visual disorders. Eur Rev Med Pharmacol Sci. 1999;3(2):71-74.

7. Torelli P, D'Amico D. An updated review of migraine and co-morbid psychiatric disorders. Neurol Sci. 2004;25(Suppl 3):S234-S235.

8. Molgat CV, Patten SB. Comorbidity of major depression and migraine - a Canadian population-based study. Can J Psychiatry. 2005;50(13):832-837.

9. Jette N, Patten S, Williams J, Becker W, Wiebe S. Comorbidity of migraine and psychiatric disorders - a national population-based study. Headache. 2008;48(4):501-516.

10. Pellock JM. Understanding co-morbidities affecting children with epilepsy. Neurology. 2004;62(5 Suppl 2):S17-S23.

11. Jan MM. History of motion sickness is predictive of childhood migraine. J Paediatr Child Health. 1998;34(5):483-484.

12. Kuritzky A, Ziegler DK, Hassanein R. Vertigo, motion sickness and migraine. Headache. 1981;21(5):227-231.

13. Rashed H, Abell TL, Familoni BO, Cardoso S. Autonomic function in cyclic vomiting syndrome and classic migraine. Dig Dis Sci. 1999;44 (8 Suppl):74S-78S.

14. Dodick DW, Eross EJ, Parish JM, Silber M. Clinical, anatomical, and physiologic relationship between sleep and headache. Headache. 2003;43(3):282-292.

15. Raskin NH, Knittle SC. Ice cream headache and orthostatic symptoms in patients with migraine. Headache. 1976;16(5):222-225.

16. Montagna P. The physiopathology of migraine: the contribution of genetics. Neurol Sci. 2004;25(Supp1 3):S93-S96.

17. Headache Classification Subcommittee of the International Headache Society. The International Classification of Headache Disorders: 2nd edition. Cephalalgia. 2004;24(Suppl 1):9-160.

18. Drigo $P$, Carli G, Laverda AM. Benign paroxysmal torticollis of infancy. Brain Dev. 2000;22(3):169-172.

19. Giffin NJ, Benton S, Goadsby PJ. Benign paroxysmal torticollis of infancy: four new cases and linkage to CACNA1A mutation. Dev Med Child Neurol. 2002;44(7):490-493.

20. Shevell MI. Acephalgic migraines of childhood. Pediatr Neurol. 1996;14(3):211-215.

21. Lewis D, Ashwal S, Hershey A, Hirtz D, Yonker M, Silberstein S. Practice parameter: pharmacological treatment of migraine headache in children and adolescents: report of the American Academy of Neurology Quality Standards Subcommittee and the Practice Committee of the Child Neurology Society. Neurology. 2004;63(12):2215-2224.

22. Sartory G, Muller B, Metsch J, Pothmann R. A comparison of psychological and pharmacological treatment of pediatric migraine. Behav Res Ther. 1998;36(12):1155-1170.

23. Linder SL, Mathew NT, Cady RK, Finlayson G, Ishkanian G, Lewis DW. Efficacy and tolerability of almotriptan in adolescents: a randomized, double-blind, placebo-controlled trial. Headache. 2008;48(9):1326-1336.

24. Schoenen J, Jacquy J, Lenaerts M. Effectiveness of high-dose riboflavin in migraine prophylaxis. A randomized controlled trial. Neurology. 1998;50(2):466-470.
25. Lewis D, Winner P, Saper J, et al. Randomized, double-blind, placebo-controlled study to evaluate the efficacy and safety of topiramate for migraine prevention in pediatric subjects 12 to 17 years of age. Pediatrics. 2009;123(3):924-934.

26. Kavuk I, Yavuz A, Cetindere U, Agelink MW, Diener HC. Epidemiology of chronic daily headache. Eur J Med Res. 2003;8(6):236-240.

27. Gladstein J, Holden EW, Peralta L, Raven M. Diagnoses and symptom patterns in children presenting to a pediatric headache clinic. Headache. 1993;33(9):497-500.

28. Hershey AD, Powers SW, Bentti AL, LeCates S, deGrauw TJ. Characterization of chronic daily headaches in children in a multidisciplinary headache center. Neurology. 2001;56(8):1032-1037.

29. Siberstein SD, Lipton RB, Solomon S, Mathew NT. Classification of daily and near-daily headaches: proposed revisions to the IHS criteria. Headache. 1994;34(1):1-7.

30. Silberstein SD, Lipton RB, Sliwinski M. Classification of daily and near-daily headaches: field trial of revised IHS criteria. Neurology. 1996;47(4):871-875.

31. Gladstein J, Holden EW. Chronic daily headache in children and adolescents: a 2-year prospective study. Headache. 1996;36(6):349-351.

32. Mack KJ. What incites new daily persistent headache in children? Pediatr Neurol. 2004;31(2):122-125.

33. Practice parameter: the utility of neuroimaging in the evaluation of headache in patients with normal neurologic examinations (summary statement). Report of the Quality Standards Subcommittee of the American Academy of Neurology. Neurology. 1994;44(7):1353-1354.

34. Hershey AD, Powers SW, Vockell AL, LeCates S, Kabbouche MA, Maynard MK. PedMIDAS: development of a questionnaire to assess disability of migraines in children. Neurology. 2001;57(11):2034-2039.

35. Mathew NT, Kurman R, Perez F. Drug induced refractory headache clinical features and management. Headache. 1990;30(10):634-638.

36. Maizels M, Burchette R. Somatic symptoms in headache patients: the influence of headache diagnosis, frequency, and comorbidity. Headache. 2004;44(10):983-993.

37. Hershey AD, Powers SW, Bentti AL, Degrauw TJ. Effectiveness of amitriptyline in the prophylactic management of childhood headaches. Headache. 2000;40(7):539-549.

38. Saper JR, Silberstein SD, Lake AE, 3rd, Winters ME. Double-blind trial of fluoxetine: chronic daily headache and migraine. Headache. 1994;34(9):497-502.

39. Mauskop A. The use of botulinum toxin in the treatment of headaches. Curr Pain Headache Rep. 2002;6(4):320-323.

40. Raskin NH. Repetitive intravenous dihydroergotamine as therapy for intractable migraine. Neurology. 1986;36(7):995-997.

41. Schwartz TH, Karpitskiy VV, Sohn RS. Intravenous valproate sodium in the treatment of daily headache. Headache. 2002;42(6):519-522.

42. Galli F, Patron L, Russo PM, Bruni O, Ferini-Strambi L, Guidetti V. Chronic daily headache in childhood and adolescence: clinical aspects and a 4-year follow-up. Cephalalgia. 2004;24(10):850-858.

43. Mauskop A. Alternative therapies in headache. Is there a role? Med Clin North Am. 2001;85(4):1077-1084.

44. Silberstein SD. Comprehensive management of headache and depression. Cephalalgia. 1998;18 Suppl 21:50-55.

45. Wang SJ, Fuh JL, Lu SR. Chronic daily headache in adolescents: an 8-year follow-up study. Neurology. 2009;73(6):416-422.
Adolescent Health, Medicine and Therapeutics

\section{Publish your work in this journal}

Adolescent Health, Medicine and Therapeutics is an international, peer-reviewed, open access journal focusing on health, pathology, and treatment issues specific to the adolescent age group. All aspects of health maintenance, preventative measures and disease treatment interventions are addressed within the journal and practitioners from

\section{Dovepress}

all disciplines are invited to submit their work as well as healthcare researchers and patient support groups.. The manuscript management system is completely online and includes a very quick and fair peerreview system. Visit http://www.dovepress.com/testimonials.php to read real quotes from published authors. 